\title{
Prediction of CTL epitope, in silico modeling and functional analysis of cytolethal distending toxin (CDT) protein of Campylobacter jejuni
}

Arun G Ingale $1^{*}$ and Susumu Goto ${ }^{2^{*}}$

\begin{abstract}
Background: Campylobacter jejuni is a potent bacterial pathogen culpable for diarrheal disease called campylobacteriosis. It is realized as a major health issue attributable to unavailability of appropriate vaccines and clinical treatment options. As other pathogens, C. jejuni entails host cellular components of an infected individual to disseminate this disease. These host-pathogen interfaces during $C$. jejuni infection are complex, vibrant and involved in the nicking of host cell environment, enzymes and pathways. Existing therapies are trusted only on a much smaller number of drugs, most of them are insufficient because of their severe host toxicity or drugresistance phenomena. To find out remedial alternatives, the identification of new biotargets is highly anticipated. Understanding the molecules involved in pathogenesis has the potential to yield new and exciting strategies for therapeutic intervention. In this direction, advances in bioinformatics have opened up new possibilities for the rapid measurement of global changes during infection and this could be exploited to understand the molecular interactions involved in campylobacteriosis.
\end{abstract}

Methods: In this study, homology modeling, epitope prediction and identification of ligand binding sites has been explored. Further attempt to generate strapping 3D model of cytolethal distending toxin protein from C. jejuni have been described for the first time.

Results: CDT protein isolated from C. jejuni was analyzed using various bioinformatics and immuno-informatics tools including sequence and structure tools. A total of fifty five antigenic determinants were predicted and prediction results of CTL epitopes revealed that five MHC ligand are found in CDT. The three potential pocket binding site are found in the sequence that can be useful for drug designing.

Conclusions: This model, we hope, will be of help in designing and predicting novel CDT inhibitors and vaccine candidates.

Keywords: Cytolethal distending toxin (CDT), CTL epitope prediction, Campylobacter jejuni, Homology modeling

\section{Background}

Campylobacter jejuni is a prominent bacterial cause of enteric campylobacteriosis in the entire world [1]. Campylobacter is extensively distributed in poultry; nevertheless, cattle, pigs, sheep, and pet animals may also be a source of these microorganisms. This infection may be due to either eating of semi cooked meat or crosscontamination of ready-to-eat food at the time of

\footnotetext{
* Correspondence: agibiotech@gmail.com; goto@kuicr.kyoto-u.ac.jp ${ }^{1}$ Department of Biotechnology, School of Life Sciences, North Maharashtra University, Jalgaon 425001, India

${ }^{2}$ Bioinformatics Centre, Institute of Chemical Research, Kyoto University, Kyoto, Japan
}

\section{Biomed Central}

(c) 2014 Ingale and Goto; licensee BioMed Central Ltd. This is an Open Access article distributed under the terms of the Creative Commons Attribution License (http://creativecommons.org/licenses/by/2.0), which permits unrestricted use, distribution, and reproduction in any medium, provided the original work is properly credited. The Creative Commons Public Domain Dedication waiver (http://creativecommons.org/publicdomain/zero/1.0/) applies to the data made available in this article, unless otherwise stated. preparation or storage. C. jejuni-linked enterocolitis is characteristically coupled with a local acute inflammatory response that involves intestinal tissue damage [2]. The genome of $C$. jejuni has been sequenced, yet only a few prospective virulence factors produced by $C$. jejuni are well considered [3].

Cytolethal distending toxins (CDT) are a class of heterotrimeric toxins produced by $C$. jejuni and also by closely related spp., such as C. fetus, C. coli [4,5], Shigella [6] and Escherichia coli [7]. This toxin is rearward transported across the golgi complex and the endoplasmic reticulum, and afterward translocated into the nuclear 
compartment, where it applies the toxic activity [8]. The CDT comprises of three protein subunits namely CdtA, $\mathrm{CdtB}$, and $\mathrm{CdtC}$ causes progressive cellular distention with ultimate cell death and have been proposed as virulence factors in the pathogenesis of $C$. jejuni [9]. These results suggest that the CDTs are involved invasion, survival and internalization into the host cell [10-13]. Although CDT from $C$. jejuni has been studied and characterized in laboratory $[14,15]$, but research on immune responses and pathogenesis of $C$. jejuni remains unexploited.

The progress in computational methods competent of predicting immune epitopes for $\mathrm{B}$ lymphocytes and $\mathrm{T}$ lymphocytes will facilitate the viewing of pathogens for immunogenic antigens. The epitope based vaccines encourage an immune response by presenting immunogenic peptides unite to major histocompatibility complex to TCR [16]. Considering the unavailability of 3D structure of CDT, it is challenging to select proper target that would lead to predict epitope and ligand binding sites in protein. Hence, this study aims to investigate the CDT of $C$. jejuni with special focus on the structural and functional aspects through bioinformatics approach. This study has important implications on the selection of CTL epitope, a critical step in the development of vaccines.

\section{Methods}

\section{Sequence acquisition and analysis}

We have received the sequence of CDT of $C$. jejuni from the NCBI database by inserting query as "CDT $C$. jejuni". The sequence was saved in FASTA format and used for further analysis. The primary structure analysis was done by using expasy ProtParam (www.expasy.org). The secondary structure of the protein was computed using different servers like Jpred3, GOR-IV and SOPMA [17] to check the presence of alpha helix and beta plated sheets in the structure. To determine the possible function of C. jejuni, the sequence was subjected to comparative protein structure modeling in the different servers.

\section{D-Model building and validation}

Cytolethal distending toxin sequence of $C$. jejuni (CDTCJ) [EDZ32284.1] was used to develop 3D structure through homology modeling because crystal or NMR structure of the CTD protein was not available in the Protein Data Bank (PDB). The 3D structure of the CDT protein was done using a restrained-based approach in Modeller. The 3D model was generated using the ModWeb server that generates 3D models along with their confidence score (C-Score). The template selection for the homology modeling of the CDT protein was performed by submitting amino acid sequence of the target protein to ModWeb server [18]. The crystal structure of CDT from Haemophillus ducreyi (PDB ID:1SR4) was used as a template. After generating the 3D model, structure analysis and stereochemical analysis were performed using different evaluation and validation tools. The final model was validated by using SAVES online tool (http:// nihserver.mbi.ucla.edu/SAVES/). The Ramachandran plot was obtained using PROCHECK [19] and RAMPAGE [20] which helped in evaluating backbone conformation. Ramachandran plot was also used to check non-GLY residues at the disallowed regions. The verify 3D and PROSA web tool [21] was used to determine Z-scores. The ERRAT was used to predict overall quality for model and quality of the model was assured using Z- scores.

\section{Epitope prediction of protein antigens}

SEPPA (Spatial Epitope Prediction of Protein Antigens) server at the Life Science and Technology School, Tongji University, Shanghai China, (http://lifecenter. sgst.cn/seppa/) was used to predict conformational Bcell epitope.

The 3D protein structure predicted by Modeller was used as an input, each residue in the query protein will be given a score according to its neighborhood residues information. Higher score corresponds to higher probability of the residue to be involved in an epitope [22]. The default values of THRESHOLD was set at 1.80, this help to specify the epitope residues [23]. Transmembrane topology of the CDTCJ protein was checked using TMHMM online tool [24] and antigenicity of protein was checked using SVMTriP online antigen prediction server [25]. The several algorithms are available that can predict the location and binding specificity of CTL epitopes in the protein sequences. In this study, the cytotoxic T-lymphocyte epitope prediction was done using NetCTL-1.2 server [26].

\section{Sub cellular localization prediction}

The sub cellular localization of CDT was predicted using CELLO, an approach based on multi-class SVM classification system [27]. CELLO uses four types of sequence coding schemes: the amino acid composition, the dipeptide composition, the partitioned amino acid composition and the sequence composition based on the physico-chemical properties of amino acids. TargetP1.1 server was also used to predict cleavage site prediction of CDT [28].

\section{Protein interaction network mapping}

Protein-protein interactions were achieved from the STRING database [29] comprising known and predicted physical and functional protein-protein interactions. STRING in protein mode was used, and only 
interactions with high confidence levels $(>0.7)$ were kept. STRING quantitatively integrates interaction data from these sources for many organisms, and transfers information among these organisms where applicable. Network visualization was done with the Cytoscape software [30].

\section{Ligand binding sites prediction}

We used MetaPocket 2.0 server (http://metapocket.eml. org) to identify ligand-binding sites on the protein surface. The MetaPocket is a consensus method [31] developed at Technical University of Dresden and Zhejiang University jointly, in which the predicted binding sites from eight methods i.e., PASS11 (PAS), LigsiteCS (LCS), Q_SiteFinder (QSF), GHECOM (GHE), POCASA (PCS), Fpocket (FPK), SURFNET (SFN), ConCavity (CON) are combined to improve the prediction success rate.

\section{Structure comparison}

The structure comparison was executed by using DaliLite server [32].

\section{Results and discussion}

The current study was originated to perform structure based sequence analysis of the CDT protein isolated from $C$. jejuni. The protein sequence was obtained from the NCBI protein database using accession number gi| 205345645|gb|EDZ32284.1| cytolethal distending toxin [Campylobacter jejuni]. Primary structure analysis revealed that the CDT protein (268 aa) had a molecular weight of $29.94 \mathrm{kD}$ and theoretical isoelectric point (PI) 6.81. An isoelectric point indicates a negatively charged protein. The instability index (II) was 18.60 , thereby categorizes the protein as a stable. The aliphatic index appeared as 84.10 and the $\mathrm{N}$-terminus of the sequence showed the presence of $M$ (Met). The negative grand average of hydropathicity (GRAVY) of -0.061 denoted that the protein was hydrophillic. The amino acids, Asn $(\mathrm{N})$, Phe (F), Ala (A), and Leu (L), were found in high praportion in the protein. The secondary structure disclosed the presence of $8.21 \% \alpha$-helices, $4.85 \% \beta$-turns, $25.37 \%$ extended strand and $61.57 \%$ coils (Figure 1 ). Transmembrane topology of the CDTCJ protein was checked using TMHMM online tool. The TMHMM server showed that residues 23-268 presented outside region, residues 5-22 were within the transmembrane and residues 1-4 were inside the region of the protein. Hydropathy analysis of CDTCJ protein of $C$. jejuni by the TOPCONS [33], Signal P-4.0 [34] and TMHMM programs suggested the presence of only one TM helix. We therefore localized the $\mathrm{N}$ terminus of CDTCJ in the cytoplasm. A consensus predicted topology is presented in Figure 2.

The sub cellular localization of CDT was predicted using CELLO, an approach based on a two-level support vector machine (SVM) system. This server predicts sub cellular localization of protein for Gram negative bacteria by supporting vector machines based on n-peptide compositions. The CELLO output gave significant reliability for outer membrane (0.198), periplasmic (1.76) extracellular (0.803) and cytoplasmic (2.493), it indicates that the protein is cytoplasmic.

\section{Model function and validation}

To determine the possible function of CDT, the sequence was subjected to comparative protein structure modeling using the target protein sequence as query for different servers described in Methods. The modeling of CDTCJ was performed using a restrained-based approach implemented in MODWEB [35] and significant hits were obtained. A set of three models for CDT protein was constructed. The $3 \mathrm{D}$ structure of a CDTCJ protein was developed from the X-ray structure of Haemophilus ducreyi (PDB ID: 1SR4 Chain A, at $2.0 \AA$

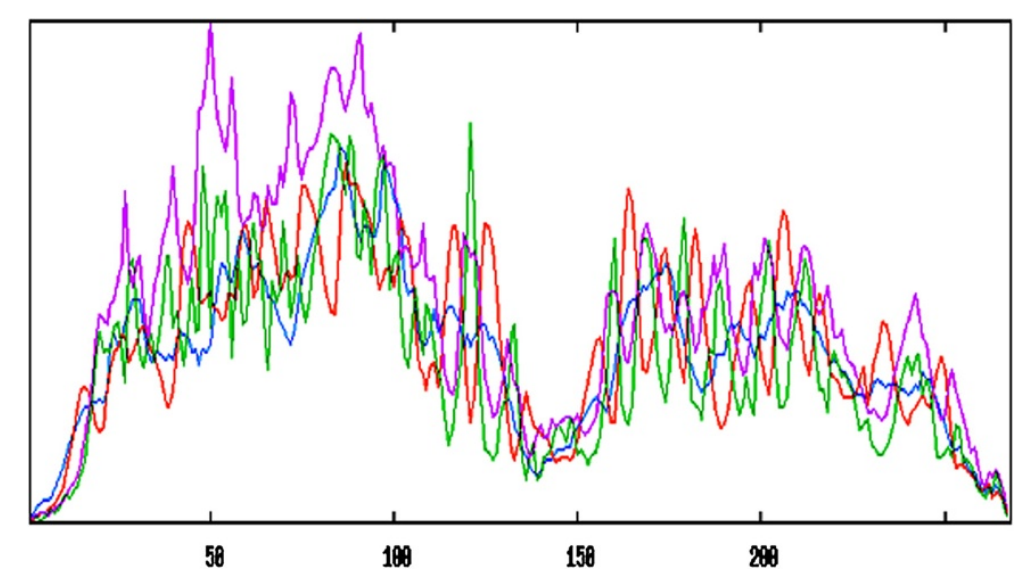

Figure 1 Secondary structure of CDT of $C$. jejuni. 


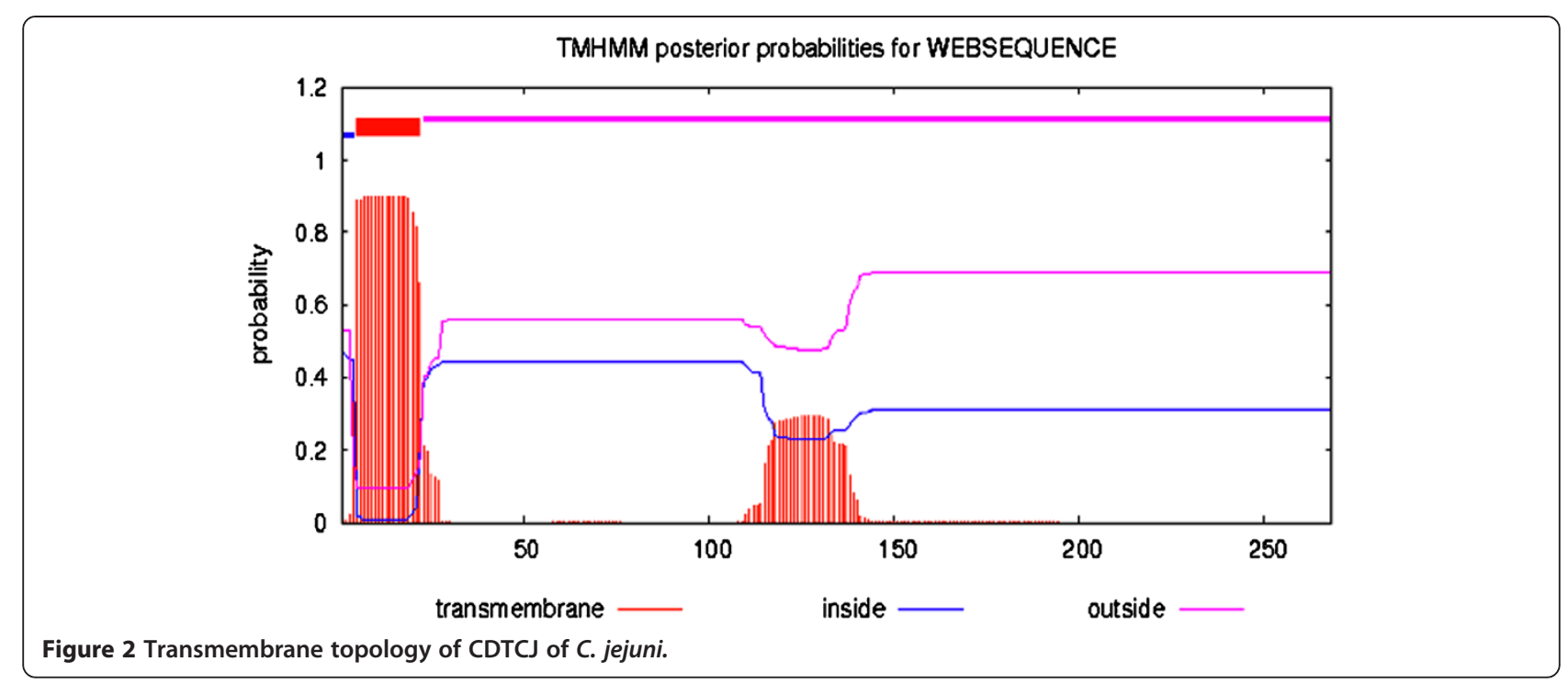

resolution) as a template for homology modeling. The alignment coverage region for target residue (113-258) showed the $37 \%$ sequence identity with template 1SR4 residue 75-219. The resulting 3D models of CDTCJ were sorted according to the scores calculated from discrete optimized protein energy (DOPE) scoring function. The final model that shared the lowest root mean square deviation (RMSD), relative to the trace (Ca atoms) of the crystal structure was selected for further studies. The validation of the model was performed by accessing the quality of backbone conformation by PROCHECK for reliability. The perceived Ramchandran plot (Psi-Phi) pairs had $86.5 \%$ of residues in most favored regions, $11.1 \%$ core residues in additional allowed regions, $1.6 \%$ residues in generously allowed regions and $0.8 \%$ residues in disallowed regions (Figure 3). These values indicated a good quality model. Whereas the crystal structure of Haemophilus ducreyi PDB ID 1SR4 shows $89 \%$ residue in most favor region [36]. To characterize the model, structural motif and mechanistically important loops were assigned to build the final 3D model of CDTCJ. The 3D model of CDTCJ using the template 1SR4, consist of two domains that encompasses $8 \beta$-sheets and $3 \alpha$-Helices (Figure 4). Verify3D and ERRAT were also used to further assess the quality of the CDTCJ model. Verify3D analyzes the compatibility

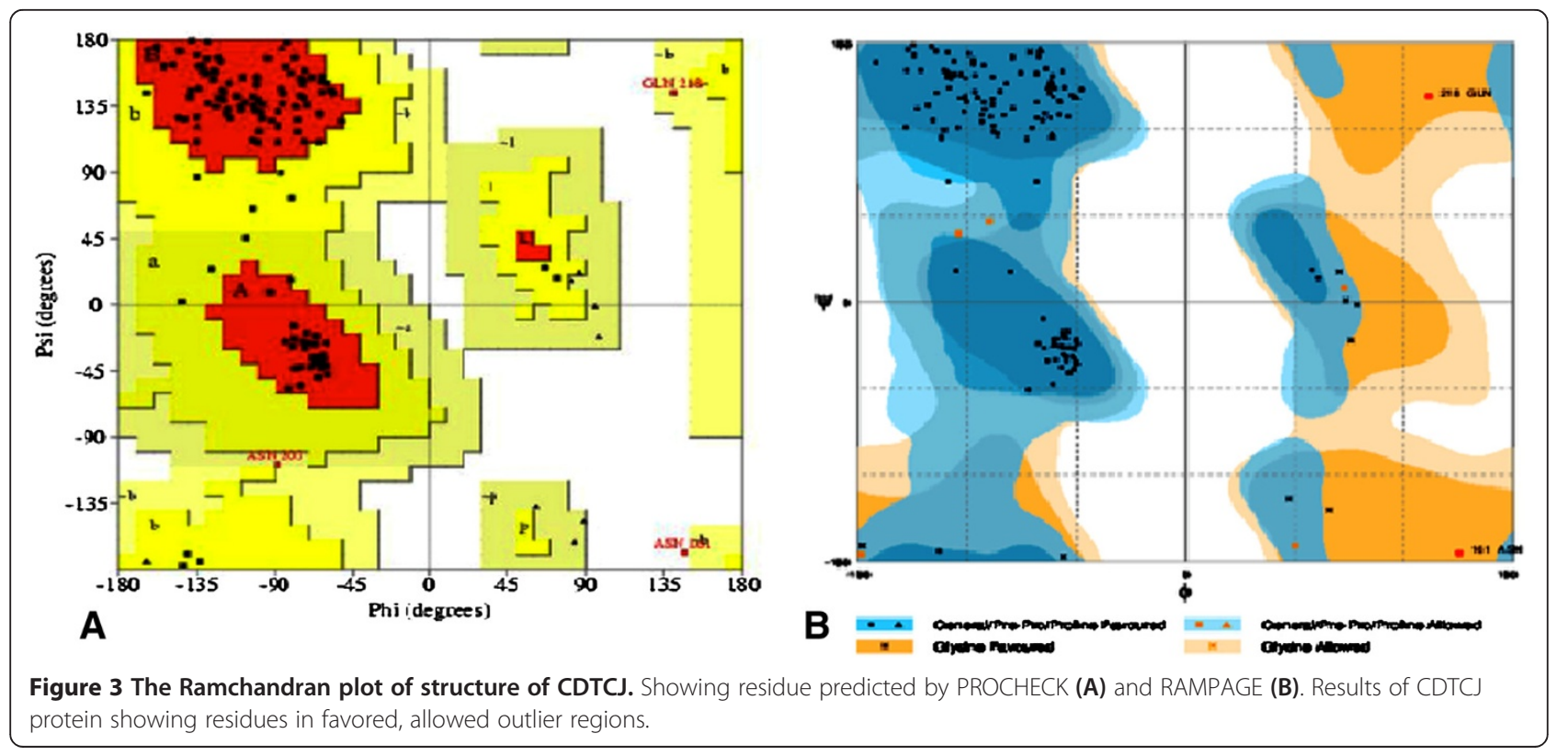




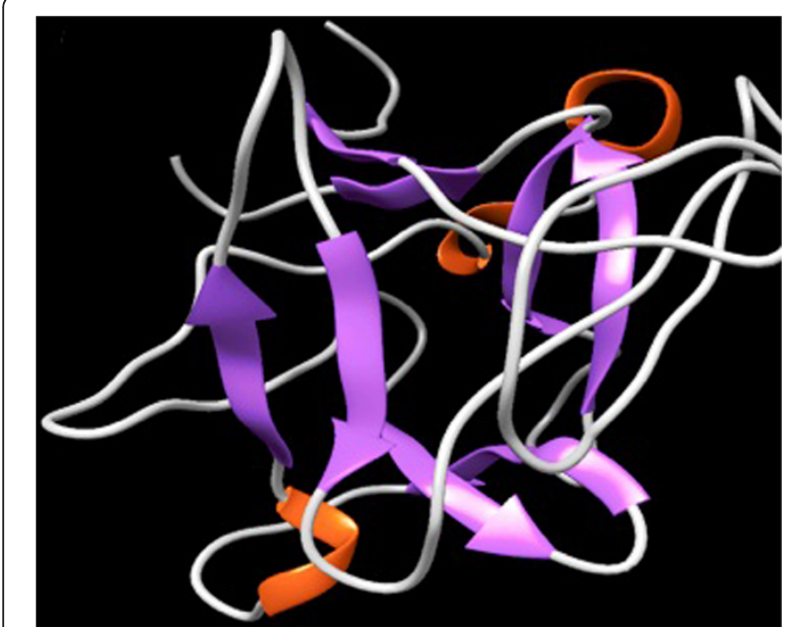

Figure 4 Homology model of CDTCJ. 3D structure of CDTCJ protein visualized by UCSF CHIMERA visualizing tool. The cartoon representation of $3 \mathrm{D}$ modeled structure of CDTCJ using PDB ID: 1SR4 shows helix (orange), sheets (purple) and loops (sky blue).

of the model against its own amino acid sequence and results revealed that $59.86 \%$ of the residue had an average $3 d-1 D$ score 0.2 . Verify3D and ProSA gave good scores for overall model quality. However, the ERRAT validation of CDTCJ model indicated regions where the calculated errors were higher than expected that decreases the overall quality score to $46.7 \%$.

\section{Structure comparison analysis}

Comparative analysis of CDTCJ structure was performed using DaliLite v.3.3. server. This server is a network service for comparing protein structure in 3D and computes optimal and suboptimal structural alignments between two protein structures. It helps in understanding the fundamental role of proteins and their functions. The structural similarity relationships among protein structures allow users to infer the functions of newly discovered proteins [37]. The final refined model of CDTCJ was superimposed with template by using DaliLite. The superimposition of model to the template is shown in Figure 5 . The result provided by DaliLite servers show the 851 alignments with compatible Z-score. The highest Z-score for structure from PDB ID: 2F2F, 1SR4 was $28.3,27.5$ and percent identity 38,37 respectively. It is interesting to note that first two high $\mathrm{Z}$-score proteins are $2 \mathrm{~F} 2 \mathrm{~F}$ and $1 \mathrm{SR} 4$, were also used for the development of model 3D structure.

\section{Epitope prediction of protein antigens}

Potentially immunogenic regions of CDTCJ were predicted by using the SEPPA server. This server analyses $3 \mathrm{D}$ structures and aims at the division of antigens surface in epitopic and non epitopic patches on the basis of different propensity scores and solvent accessibility; they all rely on training datasets comprising resolved antibody/antigen complexes [38]. A total of 55 epitopes were predicted from 146 aa using default threshold value of 1.80. The predicted epitopes visualized with JMOL in different renderings are shown in Figure 6. In this structure, tints from blue to red represent a rising antigenicity. Highlighted epitope residues were predicted and shown in red solid spheres. The prediction results are also displayed in a table and each, residue is listed sequentially. The predicted epitope residues are highlighted

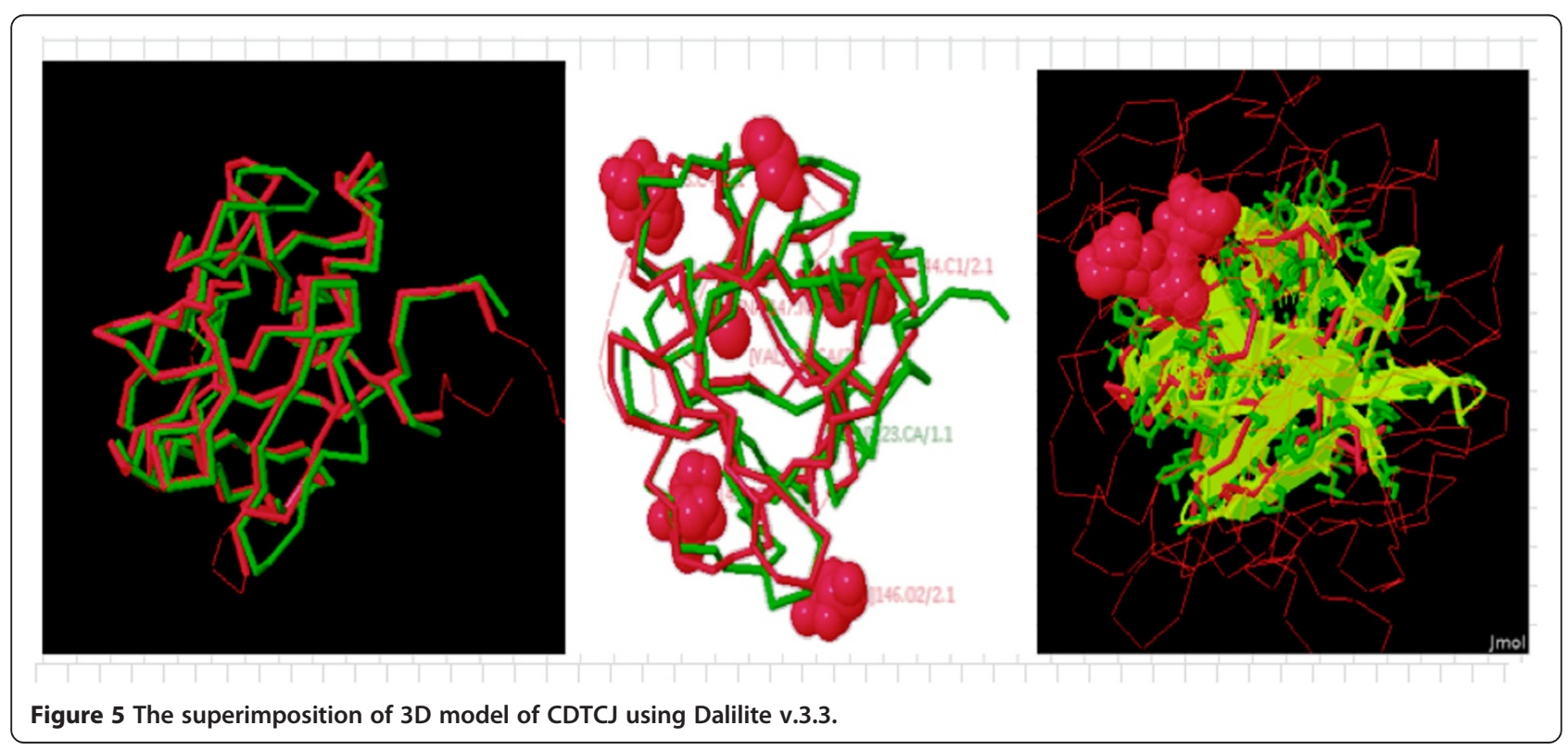




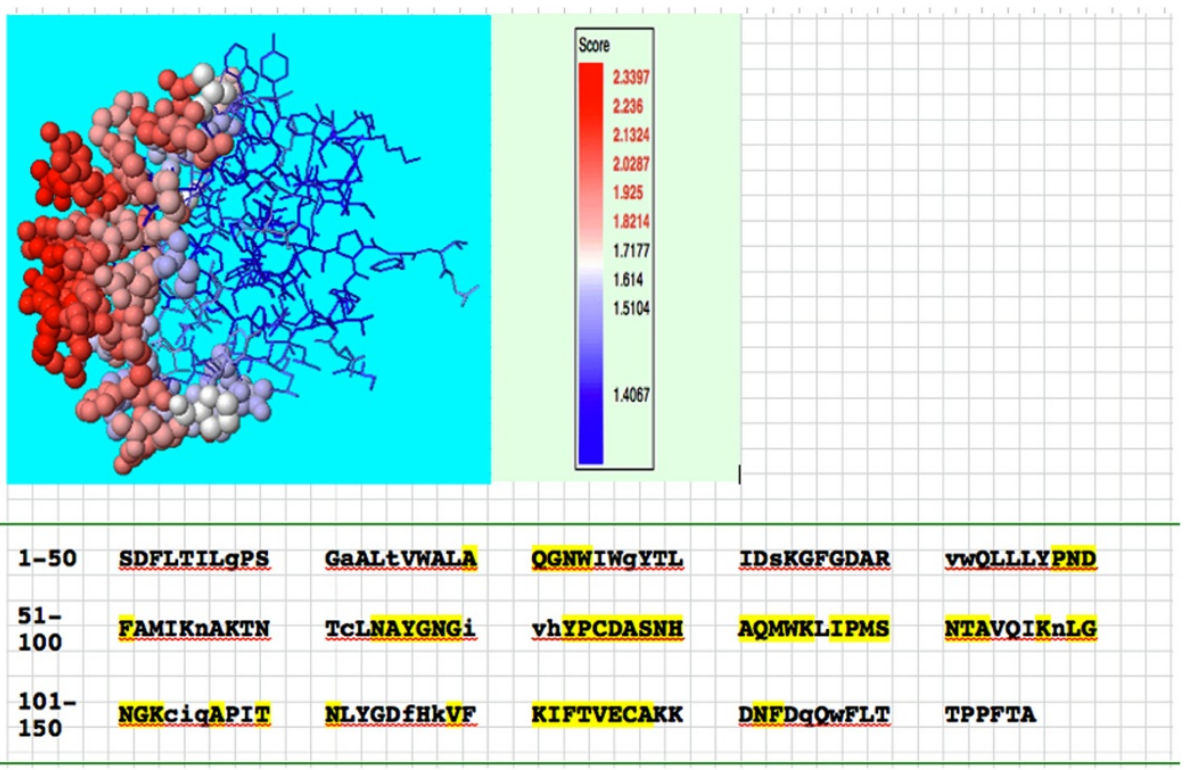

Figure 6 Antigenic epitope sites predicted by SEPPA server. The red sphere shows highest antigenicity residue and blue ones are less antigenic.

in yellow and the core residues are shown in lowercase. Antigenic epitopes that are preferentially recognized by antibodies that can help in the design of vaccine components and immuno-diagnostic reagents [39].

\section{Cytotoxic T-Lymphocytes (CTL) epitopes}

Epitope predictors are routinely tested on large sets of epitopes derived from various pathogens. Schellens et al. [40] identified eighteen new CTL epitopes out of a set of twenty two predicted CTL epitopes in HIV-1 using NetCTL. We screened all possible peptide fragments of 9aa within a particular protein, and eliminated those fragments that cannot be correctly processed by either the proteasome, TAP or the MHC class I molecules. Prediction results of CTL epitopes revealed that five MHC ligands were found in CDT sequence having high e-value score are positioned at ${ }_{10}$ CCFMTFFLY $_{18},{ }_{39} \mathrm{DT}$ DPLKLGL $_{47},{ }_{132}$ AQGNWIWGY $_{140},{ }_{170}$ KTNTCLNAY $_{178}$ and ${ }_{217}$ IQAPITNLY $_{225}$. These are the immunodominant epitopes restricted by MHC class I located arbitrarily in the protein sequence. This data indicate that CTL epitopes in CDT are randomly distributed, and this distribution is similar to those of CTL epitopes in proteins from other proteomes.

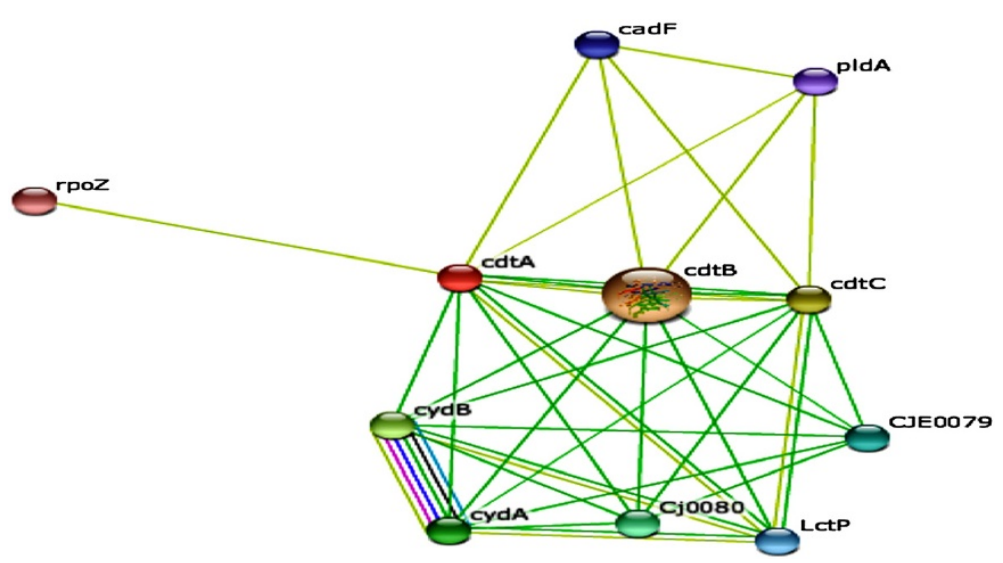

Figure 7 Interaction network of CDTCJ produced by STRING database. In this network, CDTCJ protein showed the highest interaction sCore 0.920 with CDTCJ-B protein. 
Table 1 List of predicted interactive proteins with CDTCJ of C. jejuni

\begin{tabular}{lllll}
\hline Sr. No & ID & Protein name & Amino acid residue & Score \\
\hline 1 & cdtB & Cytolethal distending toxin, subunit B & 265 & 0.920 \\
2 & cdtC & Cytolethal distending toxin, subunit C & 189 & 0.897 \\
3 & cydB & Cytochrome d ubiquinol oxidase, subunit II & 374 & 0.651 \\
4 & cydA & Cytochrome d ubiquinol oxidase, subunit I & 520 & 0.651 \\
5 & Cj0080 & Hypothetical protein & 89 & 0.651 \\
6 & Cje0079 & Hypothetical protein & 34 & 0.628 \\
7 & LctP & L lactate permease & 565 & 0.614 \\
8 & cadF & Fibronectin binding protein & 319 & 0.569 \\
9 & pldA & Phospholipase A & 329 & 0.517 \\
10 & rpoZ & DNA directed RNA polymerase, Subunit omega & 74 & 0.514 \\
\hline
\end{tabular}

\section{Protein interaction network mapping}

To compute protein-interaction properties of the CDT, we used the search tool for the retrieval of interacting genes and proteins (STRING) database of physical and functional interactions [41]. The prediction of CDTCJ interactions using protein structural similarities permit to construct various candidates interactions with possibly significant functional relevance. For this purpose, relation among the ten identified proteins was examined.
The interaction network for genetically interacting proteins possibly related in function with $C$. jejuni is shown in Figure 7, and the detail information is presented in Table 1. Green lines indicate co-localization in genomes (likely operon structures), and blue lines indicate statistically significant co-occurrence across multiple genomes. A graph of the CDTCJ network shows the identified CDTCJ-interacting proteins and phylogenomic profiling of CDT-related functions.

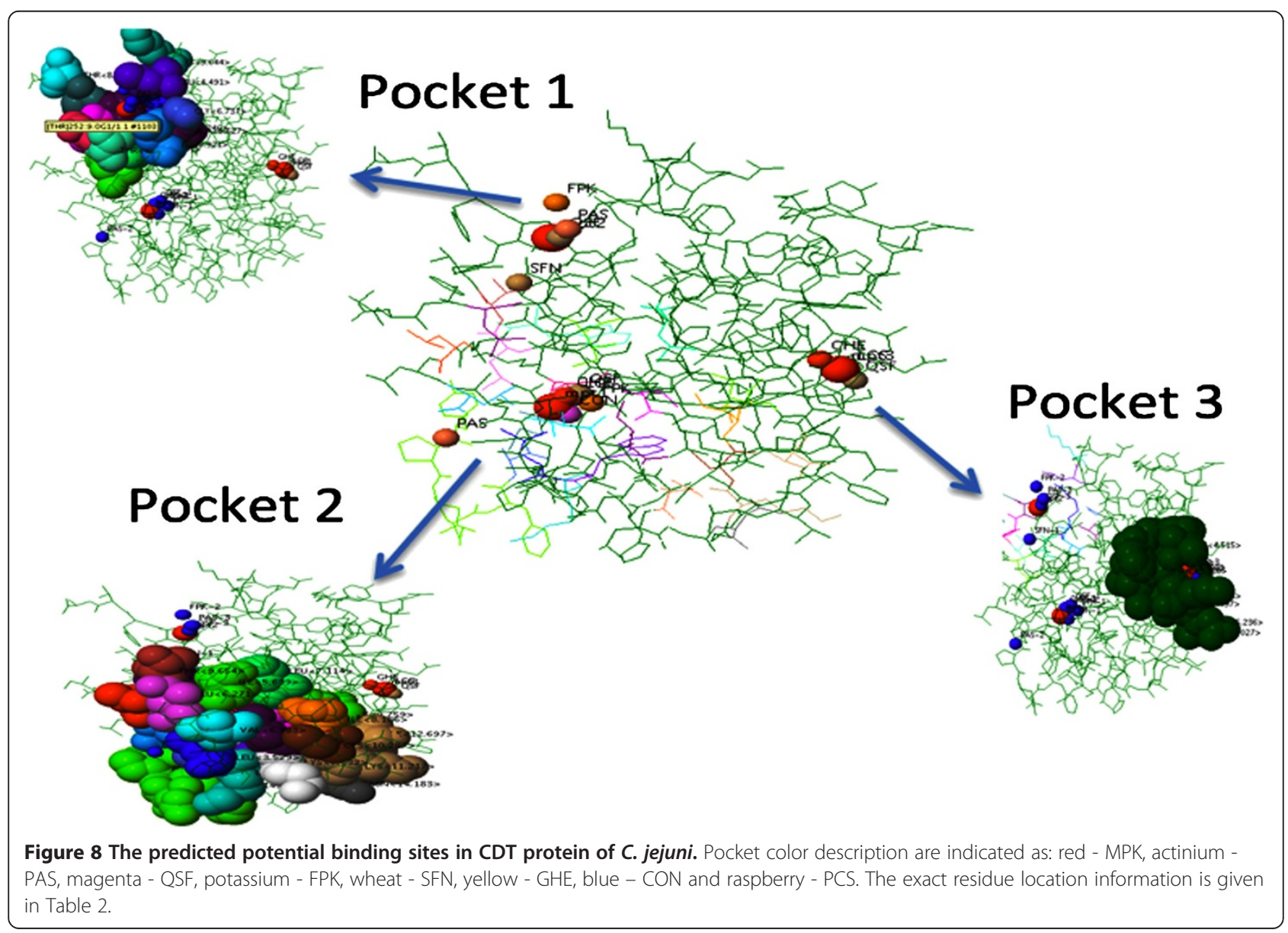


Table 2 Predicted ligand binding site in residues

\begin{tabular}{|c|c|c|c|c|c|}
\hline Site no & Residues & & & & \\
\hline \multirow[t]{6}{*}{ Header binding site 1} & ILE_9^118^ & LEU_9^126^ & TRP_9^154^ & ILE_9^166^ & LEU_9^175^ \\
\hline & ILE_9^208^ & LEU_9^116^ & TRP_9^196^ & LEU_9^198^ & LEU_9^158^ \\
\hline & ALA_9^164^ & MET_9^165^ & LYS_9^197^ & VAL_9^206^ & LEU_9^251^ \\
\hline & ILE_9^217^ & LEU_9^156^ & THR_9^252^ & ASN_9^210^ & LYS_9^215^ \\
\hline & ILE_9^234^ & CYS_9^216^ & ASN_9^213^ & LYS_9^209^ & ILE_9^182^ \\
\hline & PHE_9^163^ & ASP_9^162^ & ASN_9^161^ & TYR_9^159^ & PRO_9^160^ \\
\hline \multirow[t]{3}{*}{ Header binding site 2} & LEU_9^116^ & THR_9^117^ & THR_9^252^ & THR_9^253^ & PRO_9 ${ }^{\wedge} 254 \wedge$ \\
\hline & PRO_9^255^ & ALA_9^125^ & LEU_9^142^ & ARG_9^152^ & LEU_9^119^ \\
\hline & GLY_9^123^ & PHE_9^256^ & LYS_9^146^ & THR_9^257^ & \\
\hline \multirow[t]{2}{*}{ Header binding site 3} & TRP_9^136^ & TRP_9^138^ & VAL_9^231^ & PHE_9^232^ & ASN_9^180^ \\
\hline & GLY_9^181^ & LYS_9^233^ & GLY_9^179^ & ILE_9^182^ & ILE_9^137^ \\
\hline
\end{tabular}

\section{Ligand binding sites}

The potential binding sites (PBS) of proteins are those residues or atoms, which bind to ligands directly on protein surface; they are near to the ligand binding sites. After clustering the top three sites from different methods like PAS, QSF, FPK, SFN, GHE, CON, LCS, the MetaPocket 2.0 has predicted seven clusters for the protein structure, but we have presented here three best score pockets sites (Figure 8).

The first MetaPocket site (MPK1) consists of six pocket sites, the first pocket from GHECOM (GHE-1), the first pocket from LigisiteCS (LCS-1), the first pocket from Fpocket (FPK-1), the second pocket from PASS (PAS-2), the first pocket of Q-SiteFinder (QSF-1) and the first pocket from Concavity $(\mathrm{CON}-1)$ with total $\mathrm{Z}$ score 11.06 and size of 6 . The second MetaPocket site (MPK2) consists of four pockets, from SNF-1, FPK-2, QSF-3 and PAS-3 and the total Z-score is 7.61. The third MetaPocket site (MPK 3) consists of three pocket, from the second pocket of Q-SiteFinder (QSF-2), the third pocket from LigisiteCS (LCS-3), the third pocket from GHECOM (GHE-3) with total Z-score 2.90 and size of 3 . Table 2 shows the potential binding sites from a predicted CDT protein of $C$. jejuni in residue. The header binding sites 1,2 and 3 are designated for MetaPockets 1, 2, 3 respectively. In the case above, potential binding sites of three MetaPockets are given and they are shown in residue format with each line starting with 'RESI'. The residue described above is constructed in three parts: residue name, chain indicator and residue sequence number.

\section{Conclusions}

The purpose of the present study was to perform a global screening for new immunogenic HLA class I (HLAI) restricted cytotoxic $\mathrm{T}$ cell $(\mathrm{CTL})$ epitopes of potential utility as a vaccine candidate against campylobacteroisis. The five epitopes of CDTCJ were identified. It is anticipated that, the peptide ${ }_{170} \mathrm{KTNTCLNAY}_{178}$ can serve as novel potential vaccine candidate against diarrhea. These results have important implications for the rational design of CTL epitope-based CDT campylobacteriosis diagnostics and vaccines applicable to all ethnic groups. The presented research offered a backbone for understanding structural and functional insights of CDT protein. The additional experimental work is required to validate this epitope. The identification of ligand-binding sites is often the starting point for protein function annotation and structure-based drug design. In this study, we identify three predicted potential binding sites in CDT protein of $C$. jejuni. These are active sites on protein surface that performs protein functions.

\section{Competing interests}

The authors declare that they have no competing interest.

\section{Authors' contributions}

Al designed the study, performed in silico works and wrote the manuscript, SG provided a platform and critically reviewed the manuscript. Both authors have read and approved the final manuscript.

\section{Authors' information}

Dr. Arun Ingale (Associate Professor and Head)

Department of Biotechnology, School of Life Sciences, North Maharashtra

University, Jalgaon 425001, India.

Dr. Susumu Goto (Associate Professor)

Bioinformatics Centre, Institute of Chemical Research, Kyoto University, Kyoto, Japan.

\section{Acknowledgements}

We are thankful to the Matsumae International Foundation, Japan for providing MIF fellowship to Arun G. Ingale to pursue the above research. We also extend our gratitude towards Prof. A.B. Chaudhari who critically evaluated the manuscript.

Received: 19 September 2013 Accepted: 11 February 2014 Published: 19 February 2014

\section{References}

1. Friedman CR, Neimann J, Wegener HC, Tauxe RV: Epidemiology of Campylobacter jejuni infections in the United States and other Industrialized Nations. In Campylobacter. 2nd edition. Washington, USA: ASM International; 2000:121-138. 
2. Black RE, Levine MM, Clements ML, Hughes TP, Blaser MJ: Experimental Campylobacter jejuni infection in humans. J Infect Dis 1988, 157:472-479.

3. Parkhill J, Wren BW, Mungall K, Ketley JM, Churcher C, Basham D, Chillingworth T, Davies RM, Feltwell T, Holroyd S, Jagels K, Karlyshev AV, Moule S, Pallen MJ, Penn CW, Quail MA, Rajandream MA, Rutherford KM, van Vliet AH, Whitehead S, Barrell BG: The genome sequence of the foodborne pathogen Campylobacter jejuni reveals hyper variable sequences. Nature 2000, 403:665-668.

4. Pickett CL, Pesci EC, Cottle DL, Russell G, Erdem AN, Zeytin H: Prevalence of cytolethal distending toxin production in Campylobacter jejuni and relatedness of Campylobacter sp. cdtB genes. Infect Immun 1996, 64:2070-2078

5. Johnson WM, Lior H: Cytotoxic and cytotonic factors produced by Campylobacter jejuni, Campylobacter coli, and Campylobacter laridis. J Clin Microbiol 1986, 24:275-281.

6. Johnson WM, Lior H: Production of shiga toxin and a cytolethal distending toxin (CLDT) by serogroups of Shigella spp. FEMS Microbiol Lett 1987, 48:235-238.

7. Johnson WM, Lior H: A new heat-labile cytolethal distending toxin (CLDT) produced by Escherichia coli isolates from clinical material. Microb Pathog 1988, 4:103-113.

8. Guerra L, Cortes-Bratti X, Guidi R, Frisan T: The biology of the cytolethal distending toxins. Toxins 2011, 3:172-190

9. Walker Rl, Caldwell MB, Lee EC, Guerry P, Trust TJ, Ruiz-Palacios GM: Pathophysiology of Campylobacter enteritis. Microbiol Rev 1986, 50:81-94.

10. Biswas D, Fernando U, Reiman C, Willson P, Potter A, Allan B: Effect of cytolethal distending toxin of campylobacter jejuni on adhesion and internalization in cultured cells and in colonization of the chicken gut. Avian Dis 2006, 50:586-593.

11. Johnson WM, Lior H: A new heat-labile cytolethal distending toxin (CLDT) produced by Campylobacter spp. Microb Pathog 1988, 4:115-126.

12. Lara-Tejero M, Galan JE: CdtA, CdtB, and CdtC form a tripartite complex that is required for cytolethal distending toxin activity. Infect Immun 2001, 69:4358-4365.

13. Mao X, DiRienzo JM: Functional studies of the recombinant subunits of a cytolethal distending holotoxin. Cell Microbiol 2002, 4:245-255.

14. Wassenaar TM: Toxin production by Campylobacter spp. Clin Microbiol Rev 1997, 10:466-476.

15. Mortensen NP, Schiellerup P, Boisen N, Klein BM, Locht H, Abuoun M, Newell D, Krogfelt KA: The role of Campylobacter jejuni cytolethal distending toxin in gastroenteritis: toxin detection, antibody production, and clinical outcome. APMIS 2011, 119:626-634

16. Sing NP, Puren AJ, Bowyer SM: Sequence-based in siloco analysis of well studied Hepatitis C Virus epitopes and their variants in other genotypes (particularly genotype 5a) against South African human leukocyte antigen backgrounds. BMC Immunol 2012, 13:67-81.

17. Geourjon C, Deleage G: SOPMA: significant improvements in protein secondary structure prediction by consensus prediction from multiple alignments. Comput Appl Biosci 1995, 11:681-684.

18. Pieper U, Webb BM, Barkan DT, Schneidman-Duhovny D, Schlessinger A, Braberg $\mathrm{H}$, et al: ModBase, a database of annotated comparative protein structure models, and associated resources. Nucleic Acids Res 2011 39:D465-D474.

19. Laskowski RA, McArthur MW, Moss DS, Thornton JM: PROCHECK: a program to check the stereochemical quality of protein structures. J Appl Cryst 1993, 26:283-291

20. Lovell SC, Davis IW, Arendall WB 3rd, de Bakker PI, Word JM, Prisant MG, Richardson JS, Richardson DC: Structure validation by Calpha geometry: phi, psi and Cbeta deviation. Proteins Struct Funct Genet 2003, 50:437-450

21. Wiederstein M, Sippl M: ProSA-web: interactive web service for the recognition of errors in three-dimensional structures of proteins. Nucleic Acids Res 2007, 35:W407-W410.

22. Sweredoski MJ, Baldi P: PEPITO: improved discontinuous B-cell epitope prediction using multiple distance thresholds and half sphere exposure. Bioinformatics 2008, 24:1459-1460.

23. Sun J, Wu D, Xu T, Wang X, Xu X, et al: SEPPA: a computational server for spatial epitope prediction of protein antigens. Nucleic Acids Res 2009, 37:W612-W616.

24. Moller S, Cronning MDR, Apweiler R: Evaluation of methods for predicting membrane spanning regions. Bioinformatics 2001, 17:646-653.
25. Yao B, Zhang L, Liang S, Zhang C: SVMTriP: a method to predict antigenic epitopes using support vector machine to integrate tri-peptide similarity and propensity. PLOS ONE 2012, 7(9):e45152

26. Larsen MV, Lundegaard C, Lamberth K, Buus S, Lund O, Nielsen M: Large-scale validation of methods for cytotoxic T-lymphocyte epitope prediction. BMC Bioinforma 2007, 8:424.

27. Yu CS, Chen YC, Lu CH, Hwang JK: Prediction of protein subcellular localization. Proteins 2006, 64:643-651.

28. Emanuelsson O, Brunak S, Heijne G, Nielsen H: Locating proteins in the cell using TargetP, SignalP, and related tools. Nat Protoc 2007, 2:953-971.

29. Mering CV, Huynen M, Jaeggi D, Schmidt S, Bork P, Snel B: STRING database of predicted functional association between proteins. Nucleic Acids Res 2003, 31(1):258-261

30. Smoot M, Ono K, Ruscheinski J, Wang PL, Ideker T: Cytoscape 2.8: new features for data integration and network visualization. Bioinformatics 2011, 27(3):431-432.

31. Huang B: MetaPocket: a meta approach to improve protein ligand binding site prediction. Omics 2009, 13(4):325-330.

32. Holm L, Park J: DaliLite workbench for protein structure comparison. Bioinformatics 2000, 16(6):566-567.

33. Bernsel A, Viklund $H$, Hennerdal A, Elofsson A: TOPCONS: consensus prediction of membrane protein topology. Nucleic Acids Res 2009, 37(Webserver issue):W465-W468

34. Petersen TN, Brunak S, Heijne G, Nielsen H: SignalP 4.0: discriminating signal peptides from transmembrane regions. Nat Methods 2011, 8:785-786.

35. Eswar N, John B, Mirkovic N, Fiser A, llyin VA, Pieper U, et al: Tools for comparative protein structure modeling and analysis. Nucleic Acids Res 2003, 31:3375-3380

36. Nesic D, Hsu Y, Stenins CE: Assembly and function of a bacterial genotoxin. Nature 2004, 429:429-433.

37. Hasegawa $\mathrm{H}, \mathrm{Holm} \mathrm{L}$ : Advances and pitfalls of protein structural alignment. Curr Opin Struct Biol 2009, 19:381-389.

38. Liang S, Zheng D, Zhang C, Zacharias M: Prediction of antigenic epitopes on protein surfaces by consensus scoring. BMC Bioinforma 2009, 10:302.

39. Van Regenmortel MHV: Mapping epitope structure and activity: from one-dimensional prediction to four-dimensional description of antigenic specificity. Methods 1996, 9(3):465-472.

40. Schellensa IMM, Kesmir C, Miedemaa F, Baarlea D, Borghans JAM: An unanticipated lack of consensus cytotoxic T lymphocyte epitopes in HIV-1 databases: the contribution of prediction programs. Aids 2008, 22(1):33-37.

41. Frasnceschini A, Szklarczyk D, Frankid S, Simonovic M, et al: FranSTRING v9.1: protein-protein interaction networks, with increased coverage and integration. Nucleic Acids Res 2013, 41(Database issue):D808-D815.

doi:10.1186/1756-0500-7-92

Cite this article as: Ingale and Goto: Prediction of CTL epitope, in silico modeling and functional analysis of cytolethal distending toxin (CDT) protein of Campylobacter jejuni. BMC Research Notes 2014 7:92.

\section{Submit your next manuscript to BioMed Central and take full advantage of:}

- Convenient online submission

- Thorough peer review

- No space constraints or color figure charges

- Immediate publication on acceptance

- Inclusion in PubMed, CAS, Scopus and Google Scholar

- Research which is freely available for redistribution 